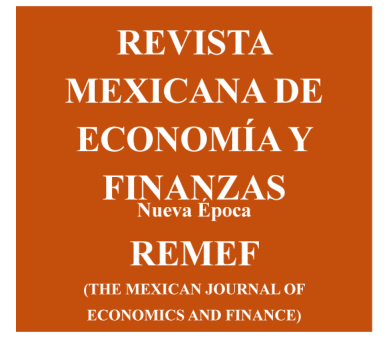
Revista Mexicana de Economía y Finanzas Nueva Época

Volumen 15 Número 1, Enero - Marzo 2020, pp. 135-144

DOI: https://doi.org/10.21919/remef.v15i1.384

(Primera recepción: 26/marzo/2019, última recepción: 5/julio/2019, aceptado: 8/octubre/2019)

\title{
Impacto de la estrategia de diversificación en el desempeño financiero en empresas de la Bolsa Mexicana de Valores
}

\author{
Eduardo Arango Herrera ${ }^{1}$ \\ Universidad Autónoma de Tamaulipas, México \\ Francisco García Fernández \\ Universidad Autónoma de Tamaulipas, México \\ José Antonio Serna Hinojosa \\ Universidad Autónoma de Tamaulipas, México
}

\section{Resumen}

El presente trabajo de investigación tiene como objetivo analizar el impacto de la diversificación mediante las métricas Herfindahl y Entropía en el desempeño financiero (EBITDA y Q-Tobins). La información fue obtenida a través de fuentes secundaras correspondientes a 133 empresas de la Bolsa Mexicana de Valores durante el periodo 2011-2016, sometiéndose a un análisis cuantitativo mediante la técnica de datos de panel. Los resultados muestran que las métricas de diversificación utilizadas resultan significativas cuando el desempeño es medido a través del EBITDA. Se recomienda observar el efecto mediante otras técnicas de análisis a través de variables intervinientes. El EBITDA puede ser una métrica utilizada para medir el desempeño de la estrategia en las empresas, siendo la principal limitación el acceso a la total información financiera de las empresas. Esta investigación propone el EBITDA como métrica de medición del desempeño financiero en un contexto de diversificación en países emergentes. Finalmente, se concluye con el efecto positivo y significativo de la métrica Herfindahl, mostrando que el EBITDA tiene mejor resultado al medir el desempeño de la diversificación que la Q-Tobins.

Clasificación JEL: L22, L25, M13

Palabras clave: Diversificación, desempeño financiero, métricas

\section{Impact of the diversification strategy on the financial performance of companies in the Mexican Stock Exchange}

\section{Abstract}

The present research work aims to analyze the impact of diversification using the Herfindahl and Entropy metrics on financial performance (EBITDA and Q-Tobins). The information was obtained through secondary sources corresponding to 133 companies of the Mexican Stock Exchange during the period 2011-2016, undergoing a quantitative analysis using the panel data technique. The results show that the diversification metrics used are significant when performance is measured through EBITDA. It is recommended to observe the effect using other analysis techniques through intervening variables. EBITDA can be a metric used to measure the performance of the strategy in companies, the main limitation being access to the total financial information of companies. This research proposes EBITDA as a metric for measuring financial performance in a context of diversification in emerging countries. Finally, it concludes with the positive and significant effect of the

\footnotetext{
${ }^{1}$ ORCID: https://orcid.org/0000-0002-3780-6783

Email: dca.arango@gmail.com

*Sin fuente de financiamiento declarada para el desarrollo de la investigación
} 


\section{Abstract}

Herfindahl metric, showing that EBITDA has a better result when measuring diversification performance than Q-Tobins.

JEL Classification: L22, L25, M13

Keywords: diversification, financial performance, metrics

\section{Introducción}

Actualmente la estrategia de diversificación ha sido considerada una de las estrategias de crecimiento organizacional más recurrente. De acuerdo con la literatura, el efecto que la estrategia de diversificación puede representar para las organizaciones no es concluyente, en algunos casos, la implementación de esta estrategia solo permite obtener beneficios al corto plazo, impidiendo que este efecto se mantenga en el largo plazo (Barney, 1991; Campillo \& Gago, 2009; Megginson et al., 2004).

La diversificación empresarial, ha sido considerada como una medida de expansión hacia otros mercados por el aumento del número de productos ofertados (Chandler, 1962). Para otros autores la diversificación es considerada como una estrategia con efectos positivos en el desempeño empresarial (Campillo \& Gago, 2008; Fernández \& Díez, 2013; López et al., 2016; Miller, 2006), otros autores establecen a la diversificación como una estrategia que representa pérdida de valor empresarial (Campillo \& Gago, 2009; Dang et al., 2016; Megginson et al., 2004; Lang \& Stulz, 1994), atribuyendo está perdida a la necesidad de mantener activas las actividades con desempeños menores.

A pesar de la amplia investigación sobre diversificación, la manera de medir la diversificación presenta confusión, sobre todo, cuando se representa con medidas cualitativas como lo son las dicotómicas (Chikoto, Ling \& Gordon, 2015; Hartzell, Sun \& Titman, 2014). En base a lo anterior y con el objetivo analizar el impacto de la diversificación mediante las métricas Herfindahl y Entropía en el desempeño financiero (EBITDA y Q-Tobins), se propone corroborar el efecto de las métricas de diversificación (Herfindahl y Entropía) sobre el desempeño financiero en un contexto de países emergentes como el mexicano, utilizando información de las empresas que cotizan en la Bolsa Mexicana de Valores (BMV) durante el periodo 2011 2016.

\section{La diversificación en el desempeño empresarial.}

Una de las características de los activos de acuerdo con la teoría de recursos y capacidades es el nivel de valía en comparación a otros activos. Esta valía es determinada por el mercado estratégico de factores de acuerdo a Barney (1991), el cual propone un conjunto de características, discutidas anteriormente, que deben poseer los activos para lograr una ventaja competitiva sostenible ante los competidores.

Trabajos establecidos como los de Ansoff (1957) y Rumelt (1974), han abordado a la diversificación, estableciendo que este tipo de estrategia ha sido un referente general para las distintas economías desarrolladas, refiriéndose como una de la estrategia dominante en actividades empresariales. Los autores (Ansoff, 1957; Rumelt, 1974) establecen dos vertientes de la diversificación i.e. relacionada y no relacionada, considerando el nivel de diversificación respecto al número de productos ofertados y los mercados en los que se encuentran posicionados.

La literatura presenta factores internos y externos que influyen en la decisión de adoptar la estrategia de diversificación. Por ejemplo, Campillo y Gago (2008) argumentan que las capacidades del CEO refuerzan positivamente la decisión de diversificar. Estos mismos autores, en otro estudio, identifican como parte de sus hallazgos algunos elementos externos (ej. intereses de deuda y poder de mercado) e internos (ej. deuda no utilizada y el uso eficiente de los activos) de la organización, que permiten generar la diversificación 
empresarial (Campillo \& Gago, 2009).

Desde una perspectiva externa, la diversificación ha sido considerada como una opción de crecimiento por parte de las empresas. La alta dirección ha considerado la concentración y la rentabilidad de la industria como los principales factores para la adopción de la estrategia de diversificación (Huerta \& Navas, 2006). En este sentido, otro factor abordado por la literatura es el estado económico del país posicionado, considerando la situación de crisis económica o desarrollo tecnológico factores que intervienen para la diversificación, incluso, para el posicionamiento de empresas provenientes del extranjero (Campillo \& Gago, 2009).

Desde una perspectiva interna, se ha estudiado el efecto del uso deficiente de activos en los rendimientos empresariales. Por ejemplo, Suárez (1993) relaciona el efecto que tienen los activos con la intención de las empresas por desarrollar nuevos productos. En su trabajo, Suárez identifica el aumento de resultados financieros provenientes del uso compartido de activos en actividades operativas, tanto para productos ya posicionados como para los nuevos. Lo anterior se ve respaldado por el estudio realizado por Fernández y Díez (2013), donde explica que a través del uso adecuado de recursos no utilizados en la generación de nuevos productos se conservaría un rendimiento positivo para la empresa.

Otro aspecto importante que une el uso de recursos y la diversificación es el uso compartido de estos, especialmente cuando las actividades de producción empresarial tienen puntos en común o complementarios en procesos (Palacio \& García, 2018). De este modo, la complementariedad de activos que se logra cuando la empresa diversifica de forma relacionada puede generar grandes beneficios para la empresa. Bettis (1981) identifica el uso de los recursos disponibles por las empresas para expandir sus líneas de productos en sectores relacionados con el producto principal.

En la literatura de diversificación se han identificado algunas métricas para dicha variable, encontrándose tanto cualitativas como cuantitativas (Berry et al., 2012; Liebenberg \& Sommer, 2008; Patrisia \& Dastgir, 2017; Robins \& Wiersema, 2003). Por un lado las cualitativas, explican las vertientes mediante la observación del posicionamiento de los productos en los sectores, mientras que por el otro lado, las cuantitativas permiten obtener un nivel mediante valores continuos, como es el caso de los índices Herfindahl y Entropía.

En un contexto latinoamericano, el uso de estas métricas, permite observar que el efecto de la diversificación ha sido positivo, en comparación a los países con economías desarrolladas (Giacomozzi, Mardones \& Pérez, 2013; Langebaek \& Ortiz, 2007; Puente \& Andrade, 2016). Los estudios, enmarcan el uso de recursos necesarios para el logro del efecto positivo de la diversificación en el desempeño financiero (EBIT, EBITDA, ROA, ROE y Q- Tobins).

Estudios realizados en México, muestra que la diversificación, en su vertiente relacionada, ha permitido que las empresas obtengan resultados positivos en el valor de mercado empresarial (Galván, García \& Serna, 2017; Ruiz y Steinwascher, 2007). Lo anterior es resultado de que la diversificación es posible contribuir con resultados positivos cuando se implementa de una forma adecuada (Gary, 2005).

Sin embargo, lo anterior son algunos de pocos estudios que se han realizado en el contexto de economías emergentes y el uso de la medición del desempeño financiero aún no ha sido del todo definido. Por lo que mediante este trabajo se pretende analizar la eficiencia de la diversificación desde las métricas Herfindahl y Entropía en el desempeño financiero (EBITDA y Q-Tobins).

\section{Definición y operacionalización de las variables.}

En este apartado, se explica el diseño metodológico el cual será utilizado para observar el impacto que presentan las métricas de diversificación sobre el desempeño financiero. Se recabo información a través de fuentes secundarias de las empresas que cotizan en la Bolsa Mexicana y Valores, por la característica que dicha información es pública, principalmente en los años 2011 al 2016. 
Con la amplia información de literatura con la que cuenta actualmente el tema de diversificación, el presente estudio pretende contribuir principalmente en la metodología de medición del efecto de la estrategia. Por lo tanto, se recabo información a través de la plataforma Bloomberg de las empresas que cotizaron en los años entre 2011 y 2016, excluyendo los años de algunas empresas que por razones distintas no cotizaron. Dicha información fue concentrada en una base de datos para ser manipulada por distintos métodos de análisis estadísticos a fines al objetivo de investigación planteado.

En la tabla 1, se muestra el descriptivo de cada una de las variables utilizadas, así mismo, la definición y la forma de operacionalización en la cual se trató las variables para el presente estudio.

Tabla 1. Definición de las Variables

\begin{tabular}{|c|c|c|c|}
\hline Variable & Nombre & Definición & Fuente de datos \\
\hline EBITDA & $\begin{array}{l}\text { Desempeño } \\
\text { Financiero }\end{array}$ & $\begin{array}{l}\text { Permite detectar de una mejor manera } \\
\text { la eficiencia en el uso de los recursos de } \\
\text { la organización, cuya información } \\
\text { financiera representa las ganancias que } \\
\text { la empresa genera antes de intereses, } \\
\text { impuestos, depreciación y amortización. }\end{array}$ & Bloomberg \\
\hline Q-Tobins & $\begin{array}{l}\text { Desempeño } \\
\text { Financiero }\end{array}$ & $\begin{array}{l}\text { Indicador financiero, el cual pretende } \\
\text { marcar un panorama respecto al valor } \\
\text { que posee una empresa. } \\
=\frac{\text { Valor de la empresa en el mercado }}{\text { Valor de la empresa en libros }}\end{array}$ & Bloomberg \\
\hline Herfindahl & $\begin{array}{c}\text { Índice } \\
\text { Herfindahl }\end{array}$ & $\begin{array}{l}\text { Es el cálculo basado en "N" segmentos } \\
\text { de negocio como la suma de los } \\
\text { cuadrados para cada segmento "i" de } \\
\text { ventas (Si). Mientras más cercano sea } \\
\text { el IH a cero, menor concentración } \\
\text { existirá. } \\
\text { *Debido a que la variable mide el nivel } \\
\text { de concentración por naturaleza, esta } \\
\text { fue tratada al inverso para mejor } \\
\text { interpretación del efecto sobre la } \\
\text { diversificación. Es decir, a mayor } \\
\text { Herfindahl, mayor diversificación. }\end{array}$ & Bloomberg \\
\hline Entropía & $\begin{array}{l}\text { Índice } \\
\text { Entropía }\end{array}$ & $\begin{array}{l}\text { Establece el grado de diversificación } \\
\text { entre los segmentos ofertados (i), a } \\
\text { través de las ventas (S) de la unidad } \\
\text { económica. Mientras más cercano sea } \\
\text { Entropía de cero, menor concentración } \\
\text { existirá. }\end{array}$ & Bloomberg \\
\hline Act & Tamaño & $\begin{array}{l}\text { Se representa el logaritmo natural del } \\
\text { valor total de los activos. }\end{array}$ & Bloomberg \\
\hline Ener - Tecnos & $\begin{array}{l}\text { Sectores } \\
\text { activos }\end{array}$ & $\begin{array}{l}\text { Segmentos en los que se encuentran } \\
\text { posicionadas las empresas en la BMV, } \\
\text { utilizando variables dummy } \\
\text { (considerando } 1 \text { si pertenecen al sector } \\
\text { y } 0 \text { si no lo hacen). }\end{array}$ & BMV \\
\hline
\end{tabular}

Fuente: Elaboración propia basado en datos proporcionados por Bloomberg. 
El EBITDA, cuya operacionalización se centra en el producto de la operación del negocio, proporciona la información financiera de las ganancias que la empresa genera antes de intereses, impuestos, depreciación y amortización (Bouwens, de Kok \& Verriest, 2019). A diferencia de la utilidad neta, la cual refleja el resultado después de partidas no operativas, el EBITDA presenta un resultado más cercano al uso eficiente de los recursos que la empresa administra a nivel operativo (Cornejo \& Díaz, 2006; Iço \& Braga, 2001; Nissim, 2017).

Para el caso del indicador Q-Tobins presenta en la literatura como un indicador que representa el desempeño pasado como el esperado en el futuro (Copeland \& Weston, 1988; Singh, et al., 2018; Zhao, Teng \& Wu, 2018). Este indicador calcula la relación del valor de mercado como el valor en libros de los activos totales. Cuando la Q-Tobins arroja valores por encima de 1, esto representa que la organización ha aumentado su valor y que se encuentra con un desempeño positivo.

En relación al índice Herfindahl, ayuda por naturaleza a explicar el nivel de concentración en el que las empresas operan en los mercados, tomando en cuenta el porcentaje de ventas de los productos respecto al total de ventas, en contraste de variables dicotómicas para establecer el tipo de diversificación (Chikoto, Ling \& Gordon, 2015; Hartzell, Sun \& Titman, 2014), el índice Herfindahl mantiene la ventaja de representar la concentración mediante una escala continua, por lo que es trabajado al inverso para su mejor interpretación y comparación con el índice de entropía (Rostami, Malekian \& Kamyabi, 2017; Karna, Schommer \& Richter, 2018).

Para el caso del índice Entropía, permite categorizar a la diversificación a través de una métrica continua basada en el nivel de ventas, similar al índice Herfindahl. A diferencia del índice Herfindahl, el índice de Entropía mide por su naturaleza el nivel de diversificación mediante su fórmula directa, sin necesidad de un tratamiento adicional (Jacquemin \& Berry, 1979).

\section{Efecto de la diversificación en el desempeño financiero.}

El análisis fue realizado de las 133 empresas que cotizaron en la Bolsa Mexicana de Valores presentes en el periodo 2011 - 2016, la información fue sometida a el software estadístico Stata, para conocer las características de las variables.

En la siguiente tabla (Tabla. 2), se muestran algunos estadísticos descriptivos como las medias, desviaciones estándar y las correlaciones entre variables, mostrando una baja correlación entre las variables independientes, así mismo se presentan algunos estadísticos descriptivos de interés. 
Tabla 2. Correlaciones parciales.

Ecuación de selección $\quad(\mathrm{N}=133)$

\begin{tabular}{|c|c|c|c|c|c|c|c|c|c|c|c|c|c|c|}
\hline Variables & Media & D.E. & 1 & 2 & 3 & 4 & 5 & 6 & 7 & 8 & 9 & 10 & 11 & 12 \\
\hline EBITDA (VD) & 14884.18 & 61075.02 & 1.00 & & & & & & & & & & & \\
\hline Q-Tobins & 2.347 & 2.245 & -.0033 & 1.00 & & & & & & & & & & \\
\hline Herfindahl (VI) & 3194.08 & 2672.375 & .1373 & .0031 & 1.00 & & & & & & & & & \\
\hline Entropía (VI) & 26.821 & 5.838 & .0837 & -.0523 & .7282 & 1.00 & & & & & & & & \\
\hline Act (VC) & 493188.1 & 3055809 & .8971 & -.0661 & .0738 & .0266 & 1.00 & & & & & & & \\
\hline Ener (VC) & .0068 & .0828 & -.0142 & -.0131 & -.0399 & -.0485 & -.0117 & 1.00 & & & & & & \\
\hline Indus (VC) & .2207 & .4149 & -.1010 & -.0021 & .1696 & .0811 & -.0807 & -.0453 & 1.00 & & & & & \\
\hline Mat (VC) & .1793 & .3838 & -.0569 & -.0335 & .1091 & .1070 & -.0647 & -.0395 & -.2505 & 1.00 & & & & \\
\hline Prod_cons (VC) & .1606 & .3673 & .0201 & .1611 & -.2522 & -.2078 & -.0121 & -.0351 & -.2228 & -.1940 & 1.00 & & & \\
\hline Salud (VC) & .3448 & .1825 & -.0429 & .0172 & -.1374 & -.1291 & -.0303 & -.0162 & -.1028 & -.0898 & -.0796 & 1.00 & & \\
\hline Telecom (VC) & .630 & .2432 & .0707 & .0556 & .2172 & .2153 & -.0318 & -.0243 & -.1541 & -.1342 & -.1193 & -.0551 & 1.00 & \\
\hline Ser_fin (VC) & .1891 & .3918 & .1923 & -.1000 & -.2114 & -.1434 & .2530 & -.0409 & -.2595 & -.2260 & -.2010 & -.0928 & -.1390 & 1.00 \\
\hline Ser_bien_nbasic (VC) & .1389 & .3460 & -.0805 & -.0637 & .0862 & .0754 & -.0611 & -.0341 & -.2165 & -.1885 & -.1677 & -.0774 & -.1159 & -.1953 \\
\hline
\end{tabular}

Fuente: Elaboración propia basada en resultados Stata.

Para dar respuesta al objetivo de la investigación, utilizamos modelos de regresión usando el método de momentos generalizados, a través de efectos aleatorios. El resultado de la prueba de Hausman $(\mathrm{X} 2=5.13, \mathrm{p}=0.7433)$ verificando que el modelo de efectos aleatorios es el más apropiado. Los problemas de heterocedasticidad y autocorrelación serial fueron revisados y detectados en los datos, por lo que el uso del método de momentos generalizados permite corregir estos problemas (Arellano \& Bond, 1991; Breusch \& Pagan. 1980; Greene, 2012; Hair, et al. 1999; Hausman, 1978; Roodman, 2015).

Cuatro modelos fueron planteados para dar respuesta al objetivo analizar el impacto de la diversificación mediante las métricas Herfindahl y Entropía en el desempeño financiero (EBITDA y Q-Tobins). Estos modelos pueden visualizarse con sus respectivas variables independientes y la diferencia en el desempeño financiero (Tabla 3 ).

Modelo 1 (Wald X2 = 1699.57, $\mathrm{p}=.001$ ) muestra el efecto que representa la diversificación a través del Herfindahl en el que menciona que ante mayor diversificación impacta positivo y significativamente en el desempeño mediante el EBITDA.

Modelo 2 (Wald X2 = 39.38, $\mathrm{p}=.001$ ) se presenta el efecto de la métrica Herfindahl en la Q-Tobins, reflejando un efecto negativo y no significativo.

Modelo 3 (Wald X2 = 1680.27, $\mathrm{p}=.001$ ) se plantea el impacto de la diversificación mediante el índice de entropía frente al desempeño representado por el EBITDA, encontrando un efecto positivo pero no significativo.

Modelo 4 (Wald X2 =40.62, p =.001) por último se considera medir con la métrica de entropía a la variable de diversificación, observando así el efecto en la Q-Tobins, resultando este efecto, negativo y no significativo como predictor del desempeño financiero. 
Tabla 3. Regresiones panel - independientes y controles.

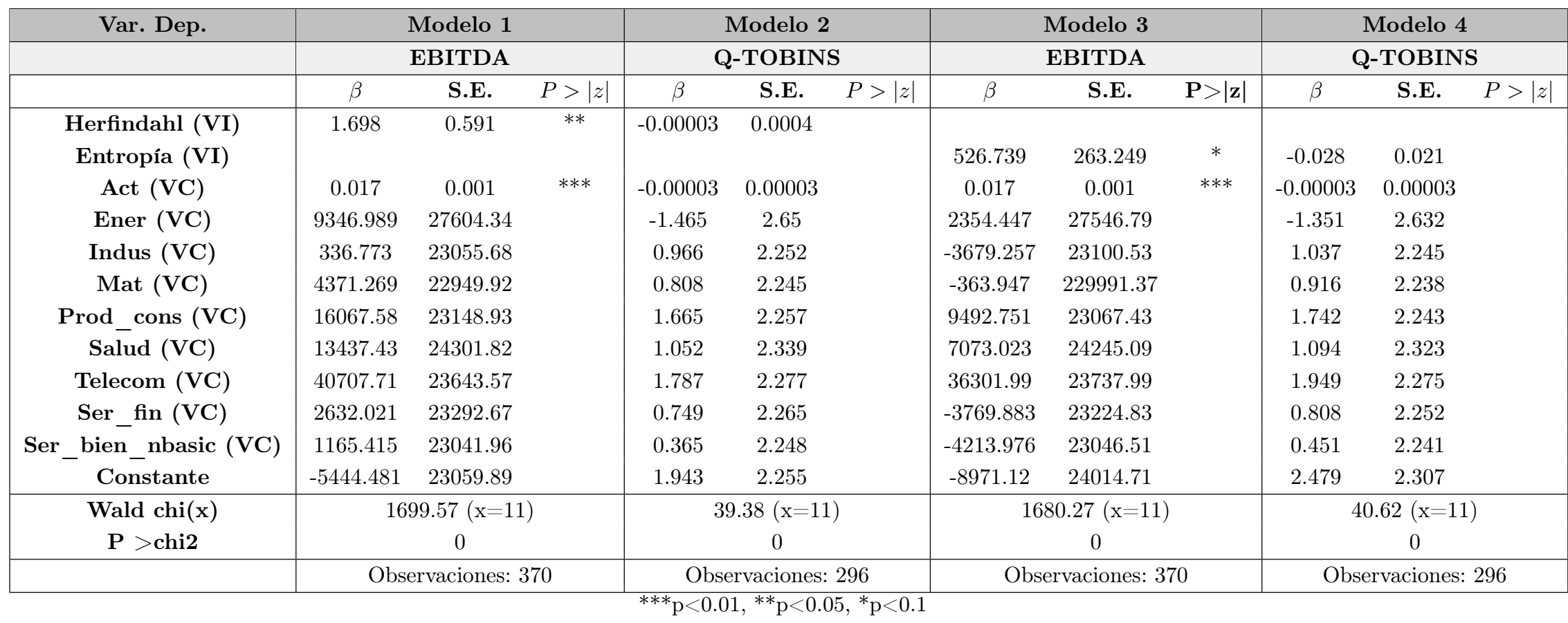

* S.E. (Estándar Error), VD (Variable Dependente), VI (Variable Independiente), VC (Variable de Control)

Fuente: Elaboración propia basada en resultados Stata. 


\section{Discusión}

El objetivo central de esta investigación es analizar el impacto de la diversificación mediante las métricas Herfindahl y Entropía en el desempeño financiero. (Ferris, Sen \& Thu, 2010; Orlitzky, Schmidt, \& Rynes, 2003; Venkatraman, \& Ramanujam, 1986). Las métricas de la diversificación implementadas tienen una gran aceptación en la literatura, ya que basan sus resultados en las ventas totales (Acar \& Sankaran, 1999; Chikoto, Ling \& Gordon, 2015; Hartzell, Sun \& Titman, 2014; Jian \& Han, 2018). Por otro lado, la medición del desempeño financiero, el uso de indicadores financieros es una herramienta muy usual (Ferris, Sen \& Thu, 2010; Orlitzky, Schmidt, \& Rynes, 2003; Venkatraman, \& Ramanujam, 1986).

Sin embargo, la literatura muestra resultados donde entre las distintas métricas para medir la diversificación, se encuentra una con mayor fortaleza que la otra para medir la diversificación frente al desempeño financiero (Chikoto, Ling \& Gordon, 2015; Jacquemin \& Berry, 1979; Schommer \& Richter, 2018). El hallazgo arrojado en la presente investigación muestra de forma empírica el efecto positivo y significativo que mantienen las métricas de diversificación (Herfindahl y Entropía) sobre la variable del desempeño financiero (EBITDA), caso contrario, se muestra un efecto no significativo cuando la variable dependiente es medida por la Q-Tobins. Los hallazgos permiten referirse, que la diversificación permite lograr efectos positivos y significativos frente al desempeño financiero, contribuyendo de este modo a la literatura sobre diversificación en el contexto empresarial mexicano.

Por lo anterior, una razón por lo que la variable EBITDA presenta efectos significativos, es a que en esta variable se encuentra un resultado proveniente directamente de las operaciones de la empresa y elimina todo aquel factor de intereses, impuestos, depreciaciones o amortizaciones que altere el resultado de implementar una estrategia u otra. Por otro lado, la variable Q-Tobins, está basado en el valor de mercado y valor en libros (Su \& Tsang, 2015; Ushijima, 2016), argumentando que el valor de mercado que se presenta en las empresas que cotizan en la BMV, es determinado por la oferta y demanda que presenta el instrumento de deuda de la misma empresa y no por el efecto del resultado de la estrategia implementada por las empresas.

\section{Conclusión}

La estrategia de diversificación en la literatura se muestra como una estrategia muy utilizada, a pesar de los cambios regulatorios en los mercados por parte del gobierno de México, sobre todo a sectores muy atractivos, donde el desempeño financiero puede reflejarse a un corto plazo, ignorando el beneficio de esta acción hacia un largo plazo directamente en la rentabilidad y la cuota de mercado.

Siguiendo el esquema de la diversificación como una estrategia sostenible, la presente investigación argumenta que la mejor forma de medir el desempeño financiero empresarial es usar la variable EBITDA, el cual, en su naturaleza, captura un escenario más puro para el resultado que trae consigo la implementación de una estrategia, lo anterior se debe, al no considerar variaciones fiscales, financieras, de amortización y depreciación.

A pesar de que en la literatura existe una discusión por el impacto que genera la diversificación, los resultados mostrados, sugieren al EBITDA como una mejor métrica para estimar el desempeño financiero empresarial sobre la Q-Tobins en el contexto mexicano, sin embargo, se propone utilizar contextos distintos para comparar los resultados. 


\section{Referencias}

[1] Acar, W., \& Sankaran, K. (1999). The myth of the unique decomposability: specializing the Herfindahl and entropy measures?. Strategic Management Journal, 20(10), 969-975.

[2] Ansoff, H. I. (1957). Strategies for diversification. Harvard business review, 35(5), 113-124.

[3] Barney, J. (1991). Firm resources and sustained competitive advantage. Journal of management, 17(1), 99-120.

[4] Berry, T. R., Liebenberg, A. P., Ruhland, J. S., y Sommer, D. W. (2012). Determinants of corporate diversification: evidence from the property-liability insurance industry. Journal of Risk and Insurance, 79(2), 381-413.

[5] Bettis, R. A. (1981). Performance differences between related and unrelated diversified firms. Strategic Mangement Journal, 2, 379-393.

[6] Bouwens, J., de Kok, T., y Verriest, A. (2019). The prevalence and validity of EBITDA as a performance measure. Comptabilité-Contrôle-Audit, 25(1), 55-105.

[7] Campillo, A., \& Gago, R. (2008). The Diversification-Performance relationship in Spanish Firms: Does The CEO'S Behaviour Style matter?. European Research Studies, 11(1/2), 57.

[8] Campillo, A., y Gago, R. (2009). ¿Qué factores determinan la decisión de diversificar? El caso de las empresas españolas (1997-2001). Investigaciones Europeas de Dirección y Economía de la Empresa, 15 (1), 15-28.

[9] Chandler, A. (1962). Strategy and structure. Massachusetts Institute of Technology Press.

[10] Chikoto, G. L., Ling, Q., \& Neely, D. G. (2016). The adoption and use of the Hirschman-Herfindahl Index in nonprofit research: Does revenue diversification measurement matter?. Voluntas: International Journal of Voluntary and Nonprofit Organizations, 27(3), 1425-1447.

[11] Chikoto, G. L., Ling, Q., \& Neely, D. G. (2016). The adoption and use of the Hirschman-Herfindahl Index in nonprofit research: Does revenue diversification measurement matter?. Voluntas: International Journal of Voluntary and Nonprofit Organizations, 27(3), 1425-1447.

[12] Copeland, T. E., Weston, J. F., \& Shastri, K. (1988). Financial theory and corporate policy. Reading, MA: Addison-Wesley, 3(1), 464-471.

[13] Cornejo, E., y Díaz Solís, D. (2006). Medidas de Ganancia: EBITDA, EBIT, Utilidad Neta y Flujo de Efectivo. Revista Economía y Administración, 1 (1) 36-40.

[14] Dang, R., Houanti, L., \& Bonnand, A. (2016). Exploring the effect of diversification strategy on R\&D intensity using quantile regression: evidence from France. Applied Economics Letters, 23(18), 1317-1320.

[15] Escobar, N., Benavides, J., \& Perafán, H., (2016). Gobierno corporativo y desempeño financiero: conceptos teóricos y evidencia empírica. Cuadernos de Contabilidad, 17(43), 203-254.

[16] Fernández, M., \& Díez, I. (2013). Effect of firm's resources on international diversification: An application in the Iberian Ham industry. European Management Journal, 31(2), 196-208.

[17] Ferris, S. P., Sen, N., \& Thu, N. T. A. (2010). Firm value and the diversification choice: international evidence from global and industrial diversification. Applied Economics Letters, 17(11), 1027-1031.

[18] Galván, A., García F., y Serna, J. (2017). Estrategia de diversificación empresarial en México: un análisis de valor. Revista Venezolana de Gerencia, 22(79), 368-386.

[19] Gary G. M. (2005). Implementation Strategy and Performance Outcomes in Related Diversification. Strategic Management Journal, vol. 26, pp. 643-664.

[20] Giacomozzi, A. M., Mardones, E. A. M., y Pérez, J. M. (2013). Relación entre el rendimiento y las estrategias de crecimiento en las sociedades anónimas chilenas. Estudios Gerenciales, 29(127), 204-212.

[21] Hartzell, J. C., Sun, L., \& Titman, S. (2014). Institutional investors as monitors of corporate diversification decisions: Evidence from real estate investment trusts. Journal of Corporate Finance, 25, 61-72.

[22] Hartzell, J. C., Sun, L., \& Titman, S. (2014). Institutional investors as monitors of corporate diversification decisions: Evidence from real estate investment trusts. Journal of Corporate Finance, 25, 61-72.

[23] Huerta, P., y Navas, J. (2006). Grado y dirección de la diversificación de las empresas industriales españolas: un análisis de la estrategia de la estrategia de diversificación relacionada. Ingeniare. Revista chilena de ingeniería, 14 (3), 213-228. 
[24] Iço, J. A., y Braga, R. P. (2001). EBITDA: lucro ajustado para fins de avaliação de desempenho operacional. Revista Contabilidade e Informação Conhecimento e Aprendizagem, Unijuí, 3(1), 39-47.

[25] Jacquemin, A. P., \& Berry, C. H. (1979). Entropy measure of diversification and corporate growth. The journal of industrial economics, 359-369.

[26] Jiang, H., \& Han, L. (2018). Does Income Diversification Benefit the Sustainable Development of Chinese Listed Banks? Analysis Based on Entropy and the Herfindahl-Hirschman Index. Entropy, 20(4), 255.

[27] Karna, A., Schommer, M., y Richter, A. (2018). Does the diversification-firm performance relationship change over time? a meta-analytical review.

[28] Lang, L., y Stulz, R. (1994). Tobin's q, corporate diversification, and firm performance. Journal of Political Economy, 102 (6), 1248-1280.

[29] Langebaek, A., y Ortiz, J. E. (2007). Q de Tobin y Gobierno corporativo de las empresas listadas en bolsa. Borradores de Economía. 447 (1).

[30] Liebenberg, A., \& Sommer, D. (2008). Effects of corporate diversification: Evidence from the property liability insurance industry. Journal of Risk and Insurance, 75(4), 893-919.

[31] López, C., Benito, S., \& Platero, M. (2016), The influence of cooperative relations on geographical expansion and diversification strategies in family firms, International Business Review.

[32] Megginson, W., Morgan, A., y Nail, L. (2004). Changes in corporate focus, ownership structure and long-run merger returns. Journal of Banking and Finance, 28 (3), 523-552.

[33] Miller, D. (2006). Technological diversity, related diversification and firm performance. Strategic Management Journal, 27 (7), 601-619.

[34] Nissim, D. (2017). Terminal Value. Columbia Business School Research Paper, 1 (1) 18-12.

[35] Orlitzky, M., Schmidt, F. L., \& Rynes, S. L. (2003). Corporate social and financial performance: A meta-analysis. Organization studies, 24(3), 403-441.

[36] Palacio, S. J. F., \& García, R. G. J. (2018). Estrategia empresarial en el sector industrial colombiano desde el enfoque de los recursos y las capacidades 2011-2012. HAL. 1(18) pp. 1-9.

[37] Patrisia, D., y Dastgir, S. (2017). Diversification and corporate social performance in manufacturing companies. Eurasian Business Review, 7(1), 121-139.

[38] Puente, M., y Andrade, F. (2016). Relación entre la diversificación de productos y la rentabilidad empresarial. Ciencia Unemi, 9(18), 73-80.

[39] Robins, J. A., \& Wiersema, M. F. (2003). The measurement of corporate portfolio strategy: Analysis of the content validity of related diversification indexes. Strategic Management Journal, 24(1), 39-59.

[40] Rostami, K.H., Malekian, E., y Kamyabi, Y. (2017). The impact of corporate diversification on firm value using entropy and herfindahl indices for the companies listed in tehran stock exchange. Risk governance \& control: financial markets \& institutions, 7(3), 77-84.

[41] Ruiz, A., \& Steinwascher, W. (2007). Corporate governance, strategic diversification and performance of firms in Mexico. MPRA Paper, 3819.

[42] Rumelt, R. (1974). Strategy, Structure and Economic Performance. Harvard Business School Press, Boston, Massachusetts.

[43] Singh, S., Tabassum, N., Darwish, T. K., \& Batsakis, G. (2018). Corporate governance and Tobin's Q as a measure of organizational performance. British Journal of Management, 29(1), 171-190.

[44] Suárez, I. (1993). Estrategia de diversificación y resultados de la empresa española. Revista de Economía Aplicada, 2(4), 103-128.

[45] Venkatraman, N., \& Ramanujam, V. (1986). Measurement of business performance in strategy research: A comparison of approaches. Academy of Management Review, 11(4), 801-814.

[46] Zhao, H., Teng, H., \& Wu, Q. (2018). The effect of corporate culture on firm performance: Evidence from China. China Journal of Accounting Research, 11(1), 1-19. 